\title{
Hardy Spaces for Quasiregular Mappings and Composition Operators
}

\author{
Tomasz Adamowicz ${ }^{1}$ (D) María J. González ${ }^{2}$
}

Received: 21 November 2020 / Accepted: 22 April 2021 / Published online: 12 May 2021

(c) The Author(s) 2021

\begin{abstract}
We define Hardy spaces $\mathcal{H}^{p}$ for quasiregular mappings in the plane, and show that for a particular class of these mappings many of the classical properties that hold in the classical setting of analytic mappings still hold. This particular class of quasiregular mappings can be characterised in terms of composition operators when the symbol is quasiconformal. Relations between Carleson measures and Hardy spaces play an important role in the discussion. This program was initiated and developed for Hardy spaces of quasiconformal mappings by Astala and Koskela in 2011 in their paper $\mathcal{H}^{p}$-theory for Quasiconformal Mappings (Pure Appl Math Q 7(1):19-50, 2011).
\end{abstract}

Keywords Carleson measure · Composition operators · Hardy spaces · Quasiregular mappings · Quasiconformal symbols

Mathematics Subject Classification $30 \mathrm{H} 10 \cdot 30 \mathrm{H} 20 \cdot 30 \mathrm{C} 20$

\section{Introduction}

Let $\mathbb{D}$ denote the unit disc $\{z \in \mathbb{C} ;|z|<1\}$ and $\mathbb{T}=\partial \mathbb{D}$. Furthermore, for a given point $\xi \in \mathbb{T}$ and $c>1$, let us denote the cone with vertex at $\xi \in \mathbb{T}$ as follows

$$
\Gamma(\xi)=\{z \in \mathbb{D} ;|z-\xi|<c(1-|z|)\}
$$

Tomasz Adamowicz

T.Adamowicz@impan.pl

María J. González

majose.gonzalez@uca.es

1 Institute of Mathematics, Polish Academy of Sciences, ul. Śniadeckich 8, 00-656 Warsaw, Poland

2 Departamento de Matemáticas, Universidad de Cádiz, 11510 Puerto Real (Cádiz), Spain 
An analytic function $f$ in $\mathbb{D}$ is in the Hardy space $\mathcal{H}^{p}$ for $0<p<\infty$, if

$$
\sup _{0<r<1} \frac{1}{2 \pi} \int_{0}^{2 \pi}\left|f\left(r e^{i \theta}\right)\right|^{p} \mathrm{~d} \theta=\|f\|_{\mathcal{H}^{p}}^{p}<\infty .
$$

The $\mathcal{H}^{p}$ theory for analytic functions is very well understood (see for instance [7], [5] or [4]). It is well known that if a function $f \in \mathcal{H}^{p}, 0<p<\infty$, then $f$ has almost everywhere non-tangential boundary limit $f\left(e^{i \theta}\right) \in L^{p}(\mathbb{T})$, and $\frac{1}{2 \pi} \int_{0}^{2 \pi}\left|f\left(e^{i \theta}\right)\right|^{p} \mathrm{~d} \theta=\|f\|_{\mathcal{H}^{p}}^{p}$. Moreover, the non-tangential maximal function of $f$, defined as $f^{*}(\xi)=\sup _{z \in \Gamma(\xi)}|f(z)|$ satisfies $f^{*} \in L^{p}(\mathbb{T})$, with $\left\|f^{*}\right\|_{L^{p}} \leq c\|f\|_{\mathcal{H}^{p}}$.

If $p \geq 2$, then by a theorem of Littlewood and Paley [11] the derivatives of an $\mathcal{H}^{p}$ function also satisfy that

$$
\int_{\mathbb{D}}\left|f^{\prime}(z)\right|^{p}(1-|z|)^{p-1} \mathrm{~d} m<\infty
$$

The aim of this note is to analyze these results in the quasiregular setting. A mapping $f: \mathbb{D} \rightarrow \mathbb{R}^{2}$ is called $K$-quasiregular for $K \geq 1$, if $f$ belongs to the Sobolev space $W_{\text {loc }}^{1,2}\left(\mathbb{D}, \mathbb{R}^{2}\right)$ and the distortion inequality

$$
|D f(z)|^{2} \leq K J f(z)
$$

holds for almost every $z \in \mathbb{D}$.

If in addition we require $f$ to be a homeomorphism, then we say that $f$ is $K$-quasiconformal. For comprehensive introductions to the topic and further references we refer, for instance, to [2], [10] and [14].

The theory of Hardy spaces for quasiconformal mappings has been developed by Astala and Koskela in their seminal paper [3]. Though their results hold also in higher dimensions, we will restrict our attention to dimension 2 only, leaving the discussion of higher dimensional cases to separate further studies.

Astala and Koskela show that many of the properties of analytic $\mathcal{H}^{p}$ functions are also shared by $\mathcal{H}^{p}$-quasiconformal maps, in particular the ones described above related to the maximal function and to the existence of boundary values in $L^{p}(\mathbb{T})$.

On the other hand, since the local integrability of $|D f|^{p}$ might fail for $K$ quasiconformal mappings when $p \geq 2 K /(K-1)$, see for instance Chapter 13 in [2], an analogue of (1) is formulated in [3] as

$$
\int_{\mathbb{D}} a_{f}(z)^{p}(1-|z|)^{p-1} \mathrm{~d} m<\infty
$$

where $a_{f}(z)=\exp \left(\frac{1}{\left|B_{z}\right|} \int_{B_{z}} \log J f^{\frac{1}{2}}(w) \mathrm{d} m\right)$ is the average derivative function, introduced by Astala and Gehring in [1]. Besides, condition (2) characterizes $\mathcal{H}^{p}$ quasiconformal mappings with no restriction on $p$ i.e. for all $0<p<\infty$ (see Theorem 5.1 in [3]). 
Note that when $f$ is conformal, $a_{f}(z)=\left|f^{\prime}(z)\right|$ due to the harmonicity of $\log \left|f^{\prime}\right|$, but that is not the case for general analytic functions. If $f$ is quasiregular, then its lack of injectivity leads to difficulties when studying $a_{f}$.

Analogous to the classical setting, we say that a quasiregular mapping $f \in \mathcal{H}_{q r}^{p}$ for a given $0<p<\infty$, if

$$
\sup _{0<r<1} \frac{1}{2 \pi} \int_{0}^{2 \pi}\left|f\left(r e^{i \theta}\right)\right|^{p} d \theta=\|f\|_{\mathcal{H}^{p}}^{p}<\infty .
$$

This class is non-empty, since any bounded quasiregular mapping belongs to $\mathcal{H}_{q r}^{p}$ for all $0<p<\infty$, because the supremum of the integral means is trivially finite.

The first part of this note is devoted to provide non-trivial examples of functions in $\mathcal{H}_{q r}^{p}$. By the Stoilow factorization theorem, any quasiregular mapping $f: \mathbb{D} \rightarrow \mathbb{R}^{2}$ can be written as $f=g \circ \phi$, where $g$ is analytic in $\mathbb{D}$ and $\phi$ is a quasiconformal mapping from $\mathbb{D}$ onto $\mathbb{D}$. Observe that, since $\phi$ is a quasiconformal selfmapping of $\mathbb{D}$, then $\phi$ has a continuous (even homeomorphic) extension to the boundary $\mathbb{T}$, see the discussion in Chapter 17 in [14], in particular [14, Theorem 17.8]. In what follows we will denote this extension still by $\phi$. Moreover, $\left.\phi\right|_{\mathbb{T}}$ is quasisymmetric.

Jerison and Weitsman constructed in [9] an example of an analytic function $g \in \mathcal{H}^{2}$ and a quasiconformal mapping $\phi$ from the disc onto itself such that the quasiregular map $f=g \circ \phi \notin \mathcal{H}_{q r}^{p}$ for any $p>0$.

In this setting we consider the composition operator $\mathcal{C}_{\phi} g=g \circ \phi$ for $g \in \mathcal{H}^{p}$, and show that under certain conditions on $\phi$, operator $C_{\phi}$ sends $\mathcal{H}^{p}$ to $\mathcal{H}_{q r}^{p}$.

Theorem 1 Let $\phi: \mathbb{D} \rightarrow \mathbb{D}$ be a quasiconformal mapping and $0<p<\infty$. Then $C_{\phi}: \mathcal{H}^{p} \rightarrow \mathcal{H}_{q r}^{p}$ is a bounded operator if and only if $\left.\phi^{-1}\right|_{\mathbb{T}}$ is a Lipschitz function.

The theorem leads to the following class of mappings: For $0<p<\infty$ we define the family of quasiregular mappings $\mathcal{F}_{p}$ as follows

$$
\mathcal{F}_{p}:=\left\{f: \mathbb{D} \rightarrow \mathbb{R}^{2} ; f=g \circ \phi, g \in \mathcal{H}^{p} \text { and }\left.\phi^{-1}\right|_{\mathbb{T}} \text { is a Lipschitzfunction }\right\}
$$

Theorem 1 shows that $\mathcal{F}_{p} \subset \mathcal{H}_{q r}^{p}$. On the other hand, it turns out that not all mappings in $\mathcal{H}_{q r}^{p}$ can be constructed this way. Indeed, an example is provided by any bounded analytic function precomposed with a quasiconformal mapping whose inverse is not Lipschitz. However, a slightly more subtle example can be given.

Theorem 2 There exists a function $f=g \circ \phi \in \mathcal{H}_{q r}^{1}$ such that $\left.\phi^{-1}\right|_{\mathbb{T}}$ is Lipschitz but $g \notin \mathcal{H}^{1}$. Moreover, such a decomposition of $f$ is unique in the sense that there are no $\tilde{g} \in \mathcal{H}^{1}, \tilde{g} \neq g$ and quasiconformal $\tilde{\phi}, \tilde{\phi} \neq \phi$ with Lipschitz $\left.\tilde{\phi}^{-1}\right|_{\mathbb{T}}$, such that $f=\tilde{g} \circ \tilde{\phi}$.

By arguing as in the proof of Theorem 1, we recover in Theorem 4 (see section on composition operators) a result for a composition operator from Bergman spaces $\mathcal{A}^{p}$ to the space of all $p$-integrable quasiregular mappings on $\mathbb{D}, \mathrm{cf}$. [6, Theorem 2].

In the second part of this note, we study the properties of $\mathcal{H}_{q r}^{p}$. One cannot expect for a general function $f \in \mathcal{H}_{q r}^{p}$ to have boundary values a.e on $\mathbb{T}$. In fact, although 
bounded planar quasiregular mappings have radial limits in a set $E$ of positive Hausdorff dimension, this set $E$ can be of arbitrarily small Hausdorff dimension (see pg. $119-120$ in [13]).

On the other hand, for quasiregular functions in $\mathcal{F}_{p}$, we show results analogous to the ones mentioned above in the context of the analytic Hardy spaces and the quasiconformal Hardy spaces.

Theorem 3 Let $0<p<\infty$ and $f$ be a $K$-quasiregular mapping in $\mathcal{F}_{p}$. Then it holds:

(i) $f \in \mathcal{H}_{q r}^{p}$.

(ii) The non-tangential boundary values $f(\xi)$ exist for a.e. $\xi \in \mathbb{T}$ and $f(\xi) \in$ $L^{p}(\mathbb{T})$.

(iii) The non-tangential maximal function $f^{*} \in L^{p}(\mathbb{T})$.

(iv) If $2 \leq p<\frac{2 K}{K-1}$, then it holds that $\int_{\mathbb{D}}|D f(z)|^{p}(1-|z|)^{p-1} \mathrm{~d} m<\infty$.

We remark that part (iv) of the theorem may fail if $0<p<2$, see [8, Theorem 1].

\section{Preliminaries}

In this note, the letter $c$ denotes a positive constant that may change at different occurrences. The notation $A \lesssim B(A \gtrsim B)$ means that there is a constant $c>0$ such that $A \leq c B(A \geq c B)$. The notation $A \simeq B$ means that $A \lesssim B \lesssim A$.

By d $m$ we denote the appropriate Lebesgue measure: 1-, 2-dimensional, depending on the context of the presentation.

Moreover, if $g$ is a complex function, then its complex derivative is denoted $g^{\prime}=$ $g_{z}$. The Jacobian of a map $f$ at a point $w$ is denoted by $J f(w)$. Note that, if $f$ is holomorphic, then $J f(w)=\left|f^{\prime}(w)\right|^{2}=\left|f_{z}(w)\right|^{2}$. If $f$ is quasiregular in the plane, then by the distortion inequality it holds that $|D f| \simeq_{c(K)}\left|f_{z}\right|$.

In what follows we often study a quasiconformal selfmapping of $\mathbb{D}$ or an analytic function in $\mathcal{H}^{p}$ which both have appropriate boundary extensions. We follow a convention to denote these extensions by the same symbols as the given quasiconformal map and the analytic function, respectively.

Finally, let us introduce the notation needed in the proof of Theorem 1. For any $z \in \mathbb{D}$, we denote by $B_{z}$ the hyperbolic ball $B_{z}=B(z, c(1-|z|))$, for some $0<c<1$, and by $I_{z}$ the interval in $\mathbb{T}$ defined as

$$
I_{z}=\left\{e^{i \theta} \in \mathbb{T}:\left|\arg \left(z e^{-i \theta}\right)\right| \leq \frac{1-|z|}{2}\right\}
$$

Note that $\left|I_{z}\right| \simeq 1-|z|$, for any $z \in \mathbb{D}$.

Moreover, if $I \subset \mathbb{T}$ is an interval, the Carleson square $S(I) \subset \mathbb{D}$ is the set

$$
S(I)=\left\{r e^{i \theta}: e^{i \theta} \in I, 1-\frac{|I|}{2 \pi} \leq r<1\right\},
$$

where $|I|$ denotes the length of the interval $I$.

The following lemma is used in proofs of Theorem 1 and part (iii) of Theorem 3 and stays that an image of a cone under a quasiconformal selfmapping of a unit disc is 
contained in a cone, whose aperture can be uniformly chosen for all boundary points. This observation is needed in order to apply the non-tangential maximal function on images of cones.

Lemma 1 Let $\phi: \mathbb{D} \rightarrow \mathbb{D}$ be a $K$-quasiconformal mapping and let $\Gamma(\xi)$ be a cone with a vertex at $\xi \in \mathbb{T}$. Then $\phi(\Gamma(\xi)) \subset \Gamma_{\phi(\xi)}$ with the aperture depending only on $K$, the aperture of $\Gamma(\xi)$ and the geometry of $\mathbb{D}$.

In the statement above we slightly abuse the term aperture, as cones in our manuscript do not necessarily have the shape of geometric cones.

Proof To simplify the notation and the argument, let us replace the unit disc by the upper half plane. Consider then a cone in the upper half plane $\mathbb{R}_{+}^{2}$, with the vertex at the origin:

$$
\Gamma=\left\{z=x+i y \in \mathbb{R}_{+}^{2}:|z|<c y\right\}
$$

for a fixed $c>1$, and let $\phi$ be a $K$-quasiconformal map from $\mathbb{R}_{+}^{2}$ onto itself with $\phi(0)=0$.

Let us show that the image of the line $L_{+}=\left\{z \in \mathbb{R}_{+}^{2}:|z|=c y, x>0\right\}$ is contained in a cone with the vertex at 0 . For that, note that the curve $\gamma=L_{+} \cup \mathbb{R}_{+}$is a quasicircle, and so is its image $\phi(\gamma)=\phi\left(L_{+}\right) \cup \mathbb{R}_{+}$.

Let $z \in L_{+}$and $w=\phi(z)$. Denote by $x_{0}$ the closest point on the real line to $w$, that is if $w=u+i v$, then $v=\left|w-x_{0}\right|$. Since the origin lies on the arc of $\phi(\gamma)$ joining $w$ and $x_{0}$, it holds by the 3 -point condition characterizing quasicircles that

$$
|w| \leq|w|+\left|x_{0}\right| \leq C\left|w-x_{0}\right|
$$

that is $|w| \leq C v$, where $C=C(K)$.

A similar argument shows that the image of the line $L_{-}=\left\{z \in \mathbb{R}_{+}^{2}:|z|=\right.$ $c y, x<0\}$ is contained in the same cone.

\section{Composition Operators with Quasiconformal Symbol}

The main goal of this section is to prove Theorem 1, also to show the boundedness of composition operator $C_{\phi}$ on Bergman spaces, see Theorem 4 below. We begin with showing the following auxiliary result used in several proofs below.

Lemma 2 Let $\phi: \mathbb{D} \rightarrow \mathbb{D}$ be $K$-quasiconformal. It holds that $\left.\phi^{-1}\right|_{\mathbb{T}}$ is Lipschitz if and only if

$$
\left|\phi^{-1}(B)\right| \leq c|B|
$$

for any hyperbolic ball $B \subset \mathbb{D}$.

Proof Denote by $w$ the center of the ball $B$, and let $\phi(z)=w$. Recall the following statement of the so-called circular distortion theorem for $K$-quasiconformal mappings 
specialized to our case of $\phi: \mathbb{D} \rightarrow \mathbb{D}$ (see e.g. [3, Lemma 2.1]): there exists a constant $C=C(K)$ so that for all $z \in \mathbb{D}$

$$
\operatorname{diam} \phi^{-1}(B) \simeq 1-|z| \text { and } \phi^{-1}(B) \supset B(z, 1 / C(1-|z|)) .
$$

Therefore, $\left|\phi^{-1}(B)\right| \simeq(1-|z|)^{2}$, and so condition (3) above reads $1-|z| \leq c(1-|w|)$.

Let $J$ be any interval on $\mathbb{T}, w_{0} \in \mathbb{D}$ be the point such that $I_{w_{0}}=J$, and $z_{0}=$ $\phi^{-1}\left(w_{0}\right)$. Then, by the circular distortion theorem, the interval $I=\phi^{-1}(J)$ contains and is contained in bounded multiples of $I_{z_{0}}$. Since $\left|I_{w_{0}}\right| \simeq 1-\left|w_{0}\right|$ and similarly for $\left|I_{z_{0}}\right|$, we can conlude that

$$
|I| \simeq 1-\left|z_{0}\right| \leq c\left(1-\left|w_{0}\right|\right) \simeq|J|
$$

that is, $\left.\phi^{-1}\right|_{\mathbb{T}}$ is Lipschitz.

In order to show the opposite implication, notice that by assuming the Lipschitz regularity of $\left.\phi^{-1}\right|_{\mathbb{T}}$, we may reverse the presented reasoning and obtain (3).

Proof of Theorem 1 Assume that $C_{\phi}: \mathcal{H}^{p} \rightarrow \mathcal{H}_{q r}^{p}$ is a bounded operator, that is for any $g \in \mathcal{H}^{p}$,

$$
\sup _{r<1} \int_{\mathbb{T}}|g(\phi(r z))|^{p} \mathrm{~d} m \leq c \int_{\mathbb{T}}|g(z)|^{p} \mathrm{~d} m .
$$

Set $g(z)=\frac{1}{(1-\bar{w} z)^{2 / p}}$, with $w \in \mathbb{D}$. Then, $g \in \mathcal{H}^{p}$, and $\|g\|_{\mathcal{H}^{p}}^{p} \simeq \frac{1}{1-|w|^{2}}$.

Next, by Fatou's lemma and (5)

$$
\begin{aligned}
\int_{\mathbb{T}} \frac{1}{|1-\bar{w} \phi(z)|^{2}} \mathrm{~d} m(z) & \leq \liminf _{r \rightarrow 1} \int_{\mathbb{T}} \frac{1}{|1-\bar{w} \phi(r z)|^{2}} \mathrm{~d} m(z) \\
& \leq \sup _{r<1} \int_{\mathbb{T}} \frac{1}{|1-\bar{w} \phi(r z)|^{2}} \mathrm{~d} m(z) \leq c \frac{1}{1-|w|^{2}}
\end{aligned}
$$

Define the measure $\mu$ on $\mathbb{T}$ as the pushforward of the Lebesgue measure on $\mathbb{T}$ via $\phi$, that is let $\mu(E):=m\left(\phi^{-1}(E)\right)$, for any set $E \subset \mathbb{T}$. Then, by the change of variables formula, (6) can be expressed in terms of $\mu$ as follows

$$
\int_{\mathbb{T}} \frac{1}{|1-\bar{w} z|^{2}} \mathrm{~d} \mu(z) \leq c \frac{1}{1-|w|^{2}} .
$$

If $z \in I_{w}$, then it holds that

$$
|1-\bar{w} z| \lesssim 1-|w| \simeq\left|I_{w}\right|
$$

Therefore, by (7) and (8) we conclude that $\mu\left(I_{w}\right) \leq c\left|I_{w}\right|$. Hence, by the definition of $\mu$

$$
\left|\phi^{-1}(I)\right| \leq c|I| \text { for any interval } I \subset \mathbb{T}
$$


as any $I=I_{w}$ for some $w \in \mathbb{D}$. This shows that function $\phi^{-1}$ is Lipschitz, cf. the proof of Lemma 2.

In order to prove the converse assertion, recall Lemma 1 and note that if $\phi^{-1}$ is Lipschitz, then by a change of variables

$$
\begin{gathered}
\sup _{r<1} \int_{\mathbb{T}}|g(\phi(r z))|^{p} \mathrm{~d} m(z) \leq \int_{\mathbb{T}}\left|(g \circ \phi)^{*}(z)\right|^{p} \mathrm{~d} m(z) \lesssim \int_{\mathbb{T}}\left|g^{*}(\phi(z))\right|^{p} \mathrm{~d} m(z) \\
\quad=\int_{\mathbb{T}}\left|g^{*}(w)\right|^{p}\left|\left(\phi^{-1}\right)^{\prime}(w)\right| \mathrm{d} m(w) \leq c \int_{\mathbb{T}}\left|g^{*}(w)\right|^{p} \mathrm{~d} m(w) \leq c\|g\|_{\mathcal{H}^{p}}^{p} .
\end{gathered}
$$

Using similar arguments, we can recover the following result (see [6, Theorem 2] for $p=2$ and Remark 1 on pg. 10 in [6] for all $0<p<\infty$ ). Recall that the Bergman space, denoted $\mathcal{A}^{p}$, consists of all holomorphic functions $p$-integrable with respect to the Lebesgue measure on $\mathbb{D}$. Furthermore, by the analogy to space $\mathcal{H}_{q r}^{p}$ we denote by $\mathcal{A}_{q r}^{p}$ the space of all $p$-integrable quasiregular mappings on $\mathbb{D}$.

Theorem 4 (FGW) The operator $C_{\phi}$ is a bounded operator from $\mathcal{A}^{p}$ to $\mathcal{A}_{q r}^{p}$ for $0<$ $p<\infty$, if and only if $\left.\phi^{-1}\right|_{\mathbb{T}}$ is Lipschitz.

Proof Recall that Carleson measures for Bergman spaces are given by the condition

$$
\mu(B) \leq c|B|, \text { for any hyperbolic ball } B \subset \mathbb{D} .
$$

Namely, Theorem 2.2 in [12] applied with $n=0, \alpha=0$ and $p=q>0$ stays that $\|g\|_{L^{p}(\mathrm{~d} \mu)} \leq C\|g\|_{\mathcal{A}^{p}}$ if and only if $\mu(B(z, r))<C r^{2}$, for all $z \in \mathbb{D}$ and $r=\frac{1}{2} \operatorname{dist}(z, \partial \mathbb{D})$. This can be shown to be equivalent to a condition $\mu(S(I))<c|I|^{2}$, for any interval $I \subset \mathbb{T}$ and the corresponding Carleson square $S(I)$.

The condition $C_{\phi}$ bounded means

$$
\int_{\mathbb{D}}|g(\phi(z))|^{p} \mathrm{~d} m \leq c\|g\|_{\mathcal{A}^{p}}^{p} .
$$

Let $\mu$ denote the pushforward measure of the Lebesgue measure on $\mathbb{D}$ under mapping $\phi$. Then, by the change of variables formula, the boundedness condition (10) can be equivalently written as

$$
\int_{\mathbb{D}}|g(z)|^{p} \mathrm{~d} \mu \leq c\|g\|_{\mathcal{A}^{p}}^{p}
$$

which in turn, by (9), holds if and only if

$$
\left|\phi^{-1}(B)\right| \leq c|B| \text { for any hyperbolic ball } B \subset \mathbb{D} .
$$

However, this by Lemma 2 is equivalent to saying that $\left.\phi^{-1}\right|_{\mathbb{T}}$ is Lipschitz. 


\section{Quasiregular Mappings $\mathcal{H}_{q r}^{p}$}

The first question we would like to address is whether any function in $\mathcal{H}_{q r}^{p}$ belongs to the family $\mathcal{F}_{p}$, see also the discussion before the statement of Theorem 2 in Introduction.

Proof of Theorem 2 Let us consider the following homeomorphism $\alpha:[-\pi, \pi] \rightarrow$ $[-\pi, \pi]$, defined as

$$
\alpha(t)= \begin{cases}\sqrt{\pi t} & 0 \leq t \leq \pi \\ -\sqrt{-\pi t} & -\pi \leq t \leq 0\end{cases}
$$

It is easy to check that the function $\phi(t)=e^{i \alpha(t)}$ is quasisymmetric on $\mathbb{T}$. Therefore, $\phi$ can be extended to a quasisymmetric mapping on the closure of $\mathbb{D}$, which will be denoted by $\phi$ as well.

Next, set $g(z)=\frac{1}{1-z}$. Then $g$ is analytic on $\mathbb{D}$ and, since $\int_{0}^{2 \pi} \mathrm{d} \theta /\left|1-e^{i \theta}\right|$ diverges, $g \notin \mathcal{H}^{1}$. On the other hand, $f=g \circ \phi \in \mathcal{H}_{q r}^{1}$ by the following reasoning.

First, recall that by Lemma 1 cones are mapped to cones and hence $|g(\phi(r \xi))| \leq$ $g^{*}(\phi(\xi))$. Let us define $g(\xi)=\frac{1}{1-\xi}$ for $\xi \in \mathbb{T}$ with convention that $g(1):=\infty$. Then it holds that $g^{*}(\xi) \simeq|g(\xi)|$ for any $\xi \in \mathbb{T}$, because if $z \in \Gamma_{\xi}$ then $|1-\xi| \lesssim|1-z|$. Therefore,

$$
\int_{\mathbb{T}}\left|f^{*}\right| \mathrm{d} m \lesssim \int_{\mathbb{T}}\left|g^{*} \circ \phi\right| \mathrm{d} m=2 \int_{0}^{\pi}\left|g^{*}\left(e^{i \alpha(t)}\right)\right| \mathrm{d} t .
$$

We apply the change of variables $s=\alpha(t)$ and get $s \mathrm{~d} s=\frac{\pi}{2} \mathrm{~d} t$ for $0<t<\pi$. Thus,

$$
\begin{aligned}
\int_{0}^{\pi}\left|g^{*}\left(e^{i \alpha(t)}\right)\right| \mathrm{d} t & \simeq \int_{0}^{\pi}\left|g^{*}\left(e^{i s}\right)\right| s \mathrm{~d} s \simeq \int_{0}^{\pi} \frac{s}{\left|1-e^{i s}\right|} \mathrm{d} s \\
& \lesssim \int_{0}^{\pi / 2} \frac{s}{\sin s} \mathrm{~d} s+\int_{\pi / 2}^{\pi} \frac{1}{\left|1-e^{i s}\right|} \mathrm{d} s<\infty .
\end{aligned}
$$

Finally, let us address the uniqueness of the decomposition for quasiregular $f$ as in the assertion of the theorem. Suppose on the contrary, that there exist a quasiconformal $\tilde{\phi} \neq \phi$ and $\tilde{g} \in \mathcal{H}^{1}$ such that $\tilde{g} \neq g$ and $f=\tilde{g} \circ \tilde{\phi}$. Then it holds that $g \circ \phi=\tilde{g} \circ \tilde{\phi}$. Set $\Phi:=\tilde{\phi} \circ \phi^{-1}$. Then $g=\tilde{g} \circ \Phi$, and therefore $\Phi: \mathbb{D} \rightarrow \mathbb{D}$ is conformal. Since the compostion operator $C_{\Phi}$ is bounded in $\mathcal{H}^{1}$, we get that $g \in \mathcal{H}^{1}$ contradicting the first assertion of the theorem.

Notice that in the above theorem we only claim that $f \notin \mathcal{F}_{1}$, but this does not exclude that $f$ may belong to $\mathcal{F}_{p}$ for some $p \neq 1$. In fact, a classical theorem by Prawitz asserts that all conformal mappings of the unit disc belong to $\mathcal{H}^{p}$ for $0<p<\frac{1}{2}$. Hence, $g \in \mathcal{H}^{p}$ for $0<p<\frac{1}{2}$ (in fact for $0<p<1$ in our case).

Finally, we present the proof of Theorem 3.

Proof of Theorem 3 (i) It is a consequence of Theorem 1. 
(ii) Let $f=g \circ \phi$. Since $g \in \mathcal{H}^{p}$, the boundary values $g\left(e^{i \theta}\right)$ exist for every $\theta \in \mathbb{T} \backslash E$ where $E$ is a set of measure 0 . Recall further, that $\phi$ has a homeomorphic extension to $\overline{\mathbb{D}}$. Therefore, $f$ has boundary values on $\mathbb{T} \backslash \phi^{-1}(E)$. Since $\left.\phi^{-1}\right|_{\mathbb{T}}$ is Lipschitz regular, we conclude that $\left|\phi^{-1}(E)\right|=0$ and, hence, $f$ has boundary values a.e. on $\mathbb{T}$.

Furthermore, a change of variables allows us to conclude the integrability of $f$ :

$$
\int_{\mathbb{T}}|f|^{p} \mathrm{~d} m=\int_{\mathbb{T}}|g \circ \phi|^{p} \mathrm{~d} m=\int_{\mathbb{T}}|g|^{p}\left|\left(\phi^{-1}\right)^{\prime}\right| d m \leq c\|g\|_{\mathcal{H}_{p}}^{p} .
$$

(iii) Since, by Lemma 1 , the image of a cone $\Gamma(z)$ at a point $z \in \mathbb{T}$ is contained in a cone at $\phi(z)$, we have

$$
\int_{\mathbb{T}}\left|f^{*}\right|^{p} d m \lesssim \int_{\mathbb{T}}\left|g^{*} \circ \phi\right|^{p} d m=\int_{\mathbb{T}}\left|g^{*}\right|^{p}\left|\left(\phi^{-1}\right)^{\prime}\right| d m \leq c\|g\|_{\mathcal{H}_{p}}^{p} .
$$

(iv) We start by recalling Theorem 3.1 in [12], here stated for $p=q \geq 2$ and $n=1$. If $g \in \mathcal{H}^{p}$ and $\mu$ is a positive measure in $\mathbb{D}$, then

$$
\int_{\mathbb{D}}\left|g^{\prime}\right|^{p} d \mu \leq c\|g\|_{\mathcal{H}^{p}}^{p}
$$

if and only if $\mu(S) \leq c(l(S))^{1+p}$, for any Carleson square $S$.

It is easy to show that the condition on the measure can be replaced by

$$
\mu(B) \leq c r_{B}^{1+p},
$$

for any hyperbolic ball $B$ of radius $r_{B}$. This occurs when the exponent is strictly bigger than 1 .

Upon defining a measure $\mu$ as the pushforward of the measure $\left|\phi^{\prime}(z)\right|^{p}(1-|z|)^{p-1} \mathrm{~d} m(z)$, we may write

$$
\begin{aligned}
\int_{\mathbb{D}}|D f(z)|^{p}(1-|z|)^{p-1} \mathrm{~d} m(z) & \lesssim \int_{\mathbb{D}}\left|g^{\prime}(\phi(z))\right|^{p}\left|\phi^{\prime}(z)\right|^{p}(1-|z|)^{p-1} \mathrm{~d} m(z) \\
& =\int_{\mathbb{D}}\left|g^{\prime}(z)\right|^{p} \mathrm{~d} \mu(z) .
\end{aligned}
$$

Here we also use that for a $K$-quasiconformal mapping $\phi$, it holds that $|D \phi| \leq$ $c(K)\left|\phi^{\prime}\right|$. Therefore, the assertion (iv) will be proven if we show that for any hyperbolic ball $\mathrm{B}$ of radius $r_{B}$

$$
\mu(B)=\int_{\phi^{-1}(B)}\left|\phi^{\prime}(z)\right|^{p}(1-|z|)^{p-1} \mathrm{~d} m(z) \leq c r_{B}^{1+p}
$$

By the circular distortion theorem, we have that set $\phi^{-1}(B) \subset \tilde{B}$ for some hyperbolic ball $\tilde{B}$, and $\phi(\tilde{B})$ is contained in another hyperbolic ball centered at the same 
point as $B$ and radius comparable to $r_{B}$ (see [3, Lemma 2.1], also the discussion at (4) above applied for $\phi$ and $\phi^{-1}$ ). In particular, $|\phi(\tilde{B})| \leq|c B| \simeq r_{B}^{2}$.

Next, observe that for $z \in \tilde{B}=\tilde{B}\left(z_{0}, r_{\tilde{B}}\right)$ it holds that $r_{\tilde{B}} \simeq 1-|z|$. This combined with estimates on the integrability of the derivatives of a $K$-quasiconformal map (see [2, Corollary 13.2.4]) when $p<2 K /(K-1)$ gives us that

$$
\begin{aligned}
\mu(B) & \leq \int_{\tilde{B}}\left|\phi^{\prime}(z)\right|^{p}(1-|z|)^{p-1} \mathrm{~d} m(z) \\
& \simeq r_{\tilde{B}}^{p-1} \int_{\tilde{B}}\left|\phi^{\prime}(z)\right|^{p} \mathrm{~d} m(z) \\
& \leq c(K, p) r_{\tilde{B}}^{p-1}\left(\frac{|\phi(\tilde{B})|}{|\tilde{B}|}\right)^{\frac{p}{2}}|\tilde{B}| \lesssim r_{\tilde{B}}^{p-1}\left(\frac{r_{B}^{2}}{r_{\tilde{B}}^{2}}\right)^{\frac{p}{2}} r_{\tilde{B}}^{2} \lesssim r_{\tilde{B}} r_{B}^{p} .
\end{aligned}
$$

Finally, by reasoning as in the proof of Lemma 2, the fact that $\left.\phi^{-1}\right|_{\mathbb{T}}$ is Lipschitz implies that $r_{\tilde{B}} \lesssim r_{B}$, see the discussion at (3) and (4). Hence, we conclude that $\mu(B) \leq r_{B}^{p+1}$, as we wanted to show. This completes the proof of assertion (iv) of the theorem.

Acknowledgements Part of the work was conducted during the Simons semester in Geometry and analysis in function and mapping theory on Euclidean and metric measure spaces at IMPAN in fall 2019. The authors would like also to thank Pekka Koskela for many valuable conversations. Moreover, the authors would like to thank the anonymous referee for interesting comments regarding Lemma 2 and Theorem 2.

Funding T.A. was supported by a Grant of National Science Center, Poland (NCN), UMO-2017/25/B/ST1/ 01955. M.J.G. was supported by Plan Propio de la UCA 2019 and Plan Nacional I+D Grant No. MTM201785666-P, Spain. This work was partially supported by the Grant 346300 for IMPAN from the Simons Foundation and the matching 2015-2019 Polish MNiSW fund.

\section{Declarations}

Conflicts of interest/Competing interests The authors declare that they have no conflict of interests nor competing interests.

Availability of data and material Not applicable. The manuscript has no associated data.

Open Access This article is licensed under a Creative Commons Attribution 4.0 International License, which permits use, sharing, adaptation, distribution and reproduction in any medium or format, as long as you give appropriate credit to the original author(s) and the source, provide a link to the Creative Commons licence, and indicate if changes were made. The images or other third party material in this article are included in the article's Creative Commons licence, unless indicated otherwise in a credit line to the material. If material is not included in the article's Creative Commons licence and your intended use is not permitted by statutory regulation or exceeds the permitted use, you will need to obtain permission directly from the copyright holder. To view a copy of this licence, visit http://creativecommons.org/licenses/by/4.0/. 


\section{References}

1. Astala, K., Gehring, F.W.: Quasiconformal analogues of theorems of Koebe and Hardy-Littlewood. Mich. Math. J. 32, 99-107 (1985)

2. Astala, K., Iwaniec, T., Martin, G.: Elliptic Partial Differential Equations and Quasiconformal Mappings in the Plane. Princeton Mathematical Series, vol. 48. Princeton University Press, Princeton, NJ (2009)

3. Astala, K., Koskela, P.: $H^{p}$-theory for quasiconformal mappings. Pure Appl. Math. Q. 7(1), 19-50 (2011)

4. Bañuelos, R., Moore, C.: Probabilistic Behavior of Harmonic Functions. Progress in Mathematics, vol. 175, xiv+204 pp. Birkhäuser Verlag, Basel (1999)

5. Duren, P.L.: Theory of $H^{p}$ Spaces. Academic Press, New York (1970)

6. Fang, X., Guo, K., Wang, Z.: Composition Operators on the Bergman Space via Quasiconformal Mappings, Preprint (2018). arXiv:1804.5352

7. Garnett, J.B.: Bounded Analytic Functions. Academic Press, New York (1981)

8. Girela, D.: Mean growth of the derivative of certain classes of analytic functions. Math. Proc. Camb. Philos. Soc. 112(2), 335-342 (1992)

9. Jerison, D., Weitsman, A.: On the means of quasiregular and quasiconformal mappings. Proc. Am. Math. Soc. 83, 304-306 (1981)

10. Lehto, O., Virtanen, K.I.: Quasiconformal Mappings in the Plane, 2nd edn. Springer, New York (1973)

11. Littlewood, J.E., Paley, R.E.: Theorems on Fourier series and power series (II). Proc. Lond. Math. Soc. (2) 42, 52-89 (1937)

12. Luecking, D.H.: Forward and reverse Carleson inequalities for functions in Bergman spaces and their derivatives. Am. J. Math. 107, 85-111 (1985)

13. Noshiro, K.: Cluster Sets. Ergebnisse der Mathematik und ihrer Grenzgebiete. N. F., Heft 28 Springer, Berlin (1960)

14. Väisälä, J.: Lectures on n-Dimensional Quasiconformal Mappings. Lecture Notes in Mathematics, vol. 229. Springer, Berlin (1971)

Publisher's Note Springer Nature remains neutral with regard to jurisdictional claims in published maps and institutional affiliations. 